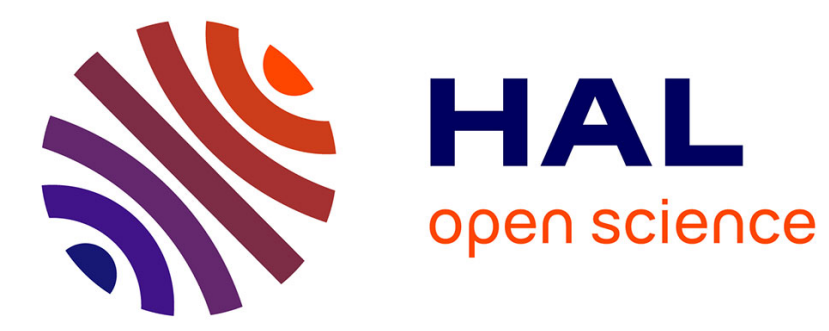

\title{
WiFi GPS based Combined positioning Algorithm
}

Soumaya Zirari, Philippe Canalda, François Spies

\section{To cite this version:}

Soumaya Zirari, Philippe Canalda, François Spies. WiFi GPS based Combined positioning Algorithm. WCNIS'10, IEEE Int. Conf. on Wireless Communications, Networking and Information Security, 2010, China. hal-00563629

\section{HAL Id: hal-00563629 \\ https://hal.science/hal-00563629}

Submitted on 7 Feb 2011

HAL is a multi-disciplinary open access archive for the deposit and dissemination of scientific research documents, whether they are published or not. The documents may come from teaching and research institutions in France or abroad, or from public or private research centers.
L'archive ouverte pluridisciplinaire HAL, est destinée au dépôt et à la diffusion de documents scientifiques de niveau recherche, publiés ou non, émanant des établissements d'enseignement et de recherche français ou étrangers, des laboratoires publics ou privés. 


\section{WiFi GPS based Combined positioning Algorithm}

\author{
Soumaya Zirari, Philippe Canalda \\ Computer Science Laboratory of the University of \\ Franche-Comté (EA 4269) \\ France \\ e-mail: soumaya.zirari@.univ-fcomte.fr, \\ philippe.canalda@univ-fcomte.fr
}

\author{
and François Spies \\ Computer Science Laboratory of the University of \\ Franche-Comté (EA 4269) \\ France \\ e-mail: francois.spies@.univ-fcomte.fr
}

\begin{abstract}
If nowadays, positioning becomes more and more accurate, and covers better and better a territory (indoor and outdoor), it remains territories where traditional (and basic) positioning system (GPS, gsm or WiFi) and hybrid ones (GPSgsm, GPS-WiFi, GPS-WiFi-gsm, ...) are insufficient and requires research investment treating combined positioning. In this paper we propose a GPS-WiFi combined positioning algorithm, based on trilateration technique. Real experiments and other simulation are conduced and demonstrate accuracy gains, even where various criteria dilution of precision (GPS dop's criteria, or ours WiFi geometrical and signal attenuation's dop proposal, or hybrid dop one's) indicate all the disruption of positioning service. A testbed scenario issued from a real urban campus environment validates not only our GPS-WiFi combined positioning algorithm but also an implementation of pertinent positioning techniques and dop's criteria. This work constitutes a further step to better position everywhere and to ensure continuity of a positioning service.
\end{abstract}

Keywords-component; positioning; GPS; GSM; WiFi; combination; hybridisation.

\section{INTRODUCTION}

Position location capabilities have become increasingly important to wireless handset manufacturers and users. New location-based applications emerged including entertainment, fleet management, commerce, safety and systems applications. The main requirement for all those new services is to provide a high accuracy independently of the environment.

The democratization of wireless and mobile networks leads to new positioning services in close environment while the emergence of new GNSS generation tends to improve the accuracy in rural and suburban areas.

Traditionally, the user position is estimated through the trilateration of at least three measured distances from fully three emitters. The distances are measured using different methods such TDOA that determines the mobile position by measuring the relative time of arrival of signals transmitted by three base stations at the handset, or the signal transmitted by a mobile at three base stations, TOA that is based on the intersection of the distance circles (the distances are calculated trough the travel time of a radio signal from a single transmitter to a remote single receiver) or AOA that allows determining the propagation direction of the signal depending on the applications and the positioning system.

In the sequel we present, first two among principal systems of positioning, the GPS system and the WiFi's one. Second we introduce new approach based on hybridization and then combination to better improve the positioning service offer. Third our contributions are described composed of an WiFi-GPS based combined positioning algorithm and a demonstrator based on innovative dilution of precision criteria that simulates and emulates real scenario of positioning only handled by such combined positioning proposal. Fourth and finally, we analyse and conclude the results obtained and laid the groundwork for future work to tackle.

\section{Global Positioning System}

The Global Positioning System [3] is a passive Global Navigation Satellite System maintained by the DoD (Department of Defence) of the United State. The GPS is the result of the merging of two independent programs that were begun in the early 1960's : the US Navy's TIMATION Program [15] and the US Air Force's 621B Project [16]. The first US pronouncement regarding civil use of GPS came in 1983. Its primary mission is to provide passive, real-time, 3$\mathrm{D}$ positioning navigation and velocity data for land, air, and sea-based applications operating anywhere in the world. The target position is measured through a range of measurements which consists in measured distances between the receiver antenna and four to ten satellites in view, and the position is determined from the adjusted intersections of the range vectors that is equivalent to trilateration solution in terrestrial surveying. These measured distances are determined by the GPS receiver by precisely measuring the time it takes a coded signal to travel from the satellites to the receiver antenna.

The fact that the user may be in environments poorly covered or not covered at all (indoor, urban canyons, etc.) makes the satellite signals reception difficult. This constraint have an important impact on the accuracy, the Quality Of Service and the service continuity that ensure that positioning can be realised in an agreed time scale and respond to a satisfactory level of service availability. 


\section{III. $\quad 802.11$}

The RADAR system [9] is a positioning system implementing a location service using the information obtained from already existing $802.11 \mathrm{WiFi}$ network. RADAR is based on Radio Frequency (RF) signal strength. In fact, RADAR uses the received signal strength indicator (RSSI) as an indicator of the distance between an AP and a receiver. This approach allows the consumer to use the positioning service without any purchase of any specialized equipment.

Based on RADAR, Ekahau emerged. Ekahau [10] Real Time Location Systems (RTLS) is a fully automated system dedicated to continually monitoring the location of assets or personnel on a campus area. Ekahau RTLS is a Wi-Fi-based location tracking solution which offers 3 to 5 meters resolution using six enterprise Wi-Fi APs. It delivers information to authorized users via the corporate network through application software or application programming interfaces in real-time. RTLS typically consists of tags, reference devices for locating tags, data network, server software and end-user application software. Ekahau RTLS uses existing Wi-Fi $(802.11 \mathrm{a} / \mathrm{b} / \mathrm{g} / \mathrm{n})$ standard access points as the reference devices for tag location and as the data network.

Other Wi-Fi positioning system is the FBCM Friis Based Calibrated Model that adapts the Friis formula which is well suited to homogeneous environments, in order to provide a better accuracy when the user is in heterogeneous environments. The FBCM[11] based on the signal strength attenuation model is dedicated to a specific building. The friis propagation model is calibrated and modified in such a way to better follow the Wi-Fi equipment mobility inside a building.

Those systems among others can be precise only if at least four Access Points (APs) are visible, which is not always the case.

\section{Hybrid Positioning Systems}

Hybrid Positioning Systems (HPS) consists in switching from one position delivered by a system to another position delivered by another system, and a position which is likely more accurate.

Skyhook Wireless developed a HPS called XPS combining the benefits of GPS, Cell Tower triangulation and Wi-Fi Positioning. The system delivers a range accuracy of $10 \mathrm{~m}-20 \mathrm{~m}$ whether indoors or outdoors, in the country or downtown [12].

Another HPS is Navizon that is a positioning system allowing the user to get its positioning information thanks to Wi-Fi and Cell Tower signals. In order to know the exact location of each Wi-Fi APs and cell towers, they need to be mapped by users with GPS enabled devices. In fact, Navizon's users with GPS enabled devices help the Navizon industry to "map the wireless landscape».

The idea of Navizon is fairly simple. The Navizon users who have GPS enabled phones, or a standalone GPS device map the location of Cell towers and Wi-Fi Access Points wherever they go, so that other users who do not have GPS will be able to use Navizon system independently of GPS.

As we can conclude, hybridization appears to be a good candidate to overcome the shortcomings of self-positioning systems but still the problem remains when no enough satellite transmitters or APs are visible, or available, to calculate the position.

\section{Combined Positioning Systems}

The main problem of the majority of the positioning systems is that not always fully three emitters are visible with strong signals and clear line of sight. Thus, manufacturers such as QUALCOMM developed the gpsOne $[5,6]$ solution for CDMA networks to address the need for accurate commercial-grade, high-availability position location technology. Traditionally, there have been two methods for providing wireless position location information, network-based solutions where the network provides to the mobile its position estimation and handsetbased solutions where a mobile node discovers its own position using some precise mechanism. GpsOne merges the two solutions and provides a chipset that triangulate measurement from cellular networks and GPS in order to estimate the user position. Other solutions proposed in [8] combine the GPS and Dead-Reckoning Navigation Systems to improve accuracy for vehicle positioning or Verizon Wireless [7] which merges the advantages of both GPS and cellular networks.

The combination offers the opportunity to address the various shortcomings that knows the positioning. Now we must think to combine different positioning systems if we are to improve the accuracy, process continuity of service or even improve the quality of service

\section{Contribution}

In this paper, we propose a combined positioning system that combines 802.11 and GPS to calculate the location. Actually, Access point have become increasingly spreading. In dense areas, different obstacles make difficult the interception of four strong signals to solve the four unknowns $(x, y, z, t)$ in a GPS positioning problem. Where $x$, $y$ and $z$ are the user coordinates and $t$ is the time difference between the GPS receiver and the satellite. We propose to complement the GPS equation system using pseudorange measurements with Signal Strength measurements from 802.11 network. In fact the aim of our proposition is if the number of visible satellites is less than four satellites, we complete the set of the equations (which is less than four equations) with equations obtained from the $\mathrm{Wi}-\mathrm{Fi}$ positioning system.

We need at least four GPS equations from four different satellites. The distance between the satellite $i$ and the user is:

$\left\{\begin{array}{l}\rho_{1}=\sqrt{\left(X^{1}-X_{u}\right)^{2}+\left(Y^{1}-Y_{u}\right)^{2}+\left(Z^{1}-Z_{u}\right)^{2}}-\delta t_{u} \\ \rho_{2}=\sqrt{\left(X^{2}-X_{u}\right)^{2}+\left(Y^{2}-Y_{u}\right)^{2}+\left(Z^{2}-Z_{u}\right)^{2}}-\delta t_{u} \\ \rho_{3}=\sqrt{\left(X^{3}-X_{u}\right)^{2}+\left(Y^{3}-Y_{u}\right)^{2}+\left(Z^{3}-Z_{u}\right)^{2}}-\delta t_{u} \\ \rho_{4}=\sqrt{\left(X^{4}-X_{u}\right)^{2}+\left(Y^{4}-Y_{u}\right)^{2}+\left(Z^{4}-Z_{u}\right)^{2}}-\delta t_{u}\end{array}\right.$ 
Where $\left(X^{i}, Y^{i}, Z^{i}\right)$ are the satellite $i$ coordinates and $\left(X_{u}, Y_{u}, Z_{u}, \delta t_{u}\right)$ are the four unknowns.

Concerning the AP equations we need at least three equations from three APs in order to estimate the user position. In fact, 3 equations are required when positioning one user outdoor, on the ground. When positoning indoor requires determining altitude, we add another equation.

$\mathrm{N}$-Dimension require $\mathrm{N}+1$-equations.

$\left\{\begin{array}{l}d_{1}=\sqrt{\left(X^{c, 1}-X_{u}\right)^{2}+\left(Y^{c, 1}-Y_{u}\right)^{2}+\left(Z^{c, 1}-Z_{u}\right)^{2}} \\ d_{2}=\sqrt{\left(X^{c, 2}-X_{u}\right)^{2}+\left(Y^{c, 2}-Y_{u}\right)^{2}+\left(Z^{c, 2}-Z_{u}\right)^{2}} \\ d_{3}=\sqrt{\left(X^{c, 3}-X_{u}\right)^{2}+\left(Y^{c, 3}-Y_{u}\right)^{2}+\left(Z^{c, 3}-Z_{u}\right)^{2}}(2)\end{array}\right.$

Where $\left(X^{c, i}, Y^{c, i}, Z^{c, i}\right)$ are the AP $i$ coordinates.
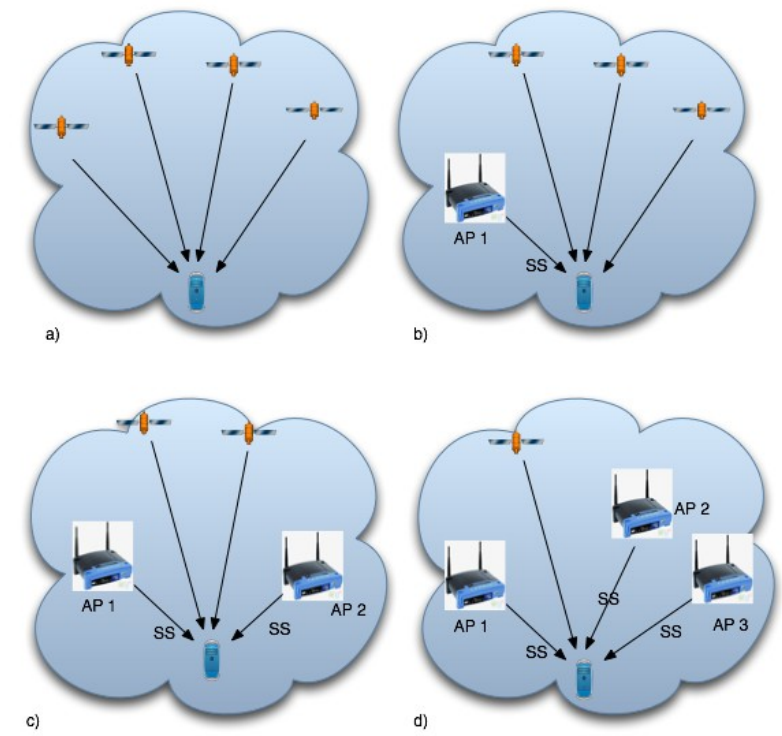

Figure 1. To estimate the user position we need at least four visible emitters from at leat one positioning system

In case we have only three visible satellites, the goal of our work is that the positioning may be without any interruption. Indeed, we complete the set of GPS equations by AP equations. In the case of three visible satellites we need supplementary AP equation. So the set of equations become as following:

$$
\left\{\begin{array}{c}
\rho_{1}=\sqrt{\left(X^{1}-X_{u}\right)^{2}+\left(Y^{1}-Y_{u}\right)^{2}+\left(Z^{1}-Z_{u}\right)^{2}}-\delta t_{u} \\
\rho_{2}=\sqrt{\left(X^{2}-X_{u}\right)^{2}+\left(Y^{2}-Y_{u}\right)^{2}+\left(Z^{2}-Z_{u}\right)^{2}}-\delta t_{u} \\
\rho_{3}=\sqrt{\left(X^{3}-X_{u}\right)^{2}+\left(Y^{3}-Y_{u}\right)^{2}+\left(Z^{3}-Z_{u}\right)^{2}}-\delta t_{u} \\
d_{1}=\sqrt{\left(X^{c, 1}-X_{u}\right)^{2}+\left(Y^{c, 1}-Y_{u}\right)^{2}+\left(Z^{c, 1}-Z_{u}\right)^{2}} \\
d_{2}=\sqrt{\left(X^{c, 2}-X_{u}\right)^{2}+\left(Y^{c, 2}-Y_{u}\right)^{2}+\left(Z^{c, 2}-Z_{u}\right)^{2}}(3)
\end{array}\right.
$$

We don't introduce the $\delta t_{u}$ in the AP equations because we consider that $d_{i}$ was obtained through Signal Strength measurements. Indeed, we don't take into account the difference time between the AP and the user.

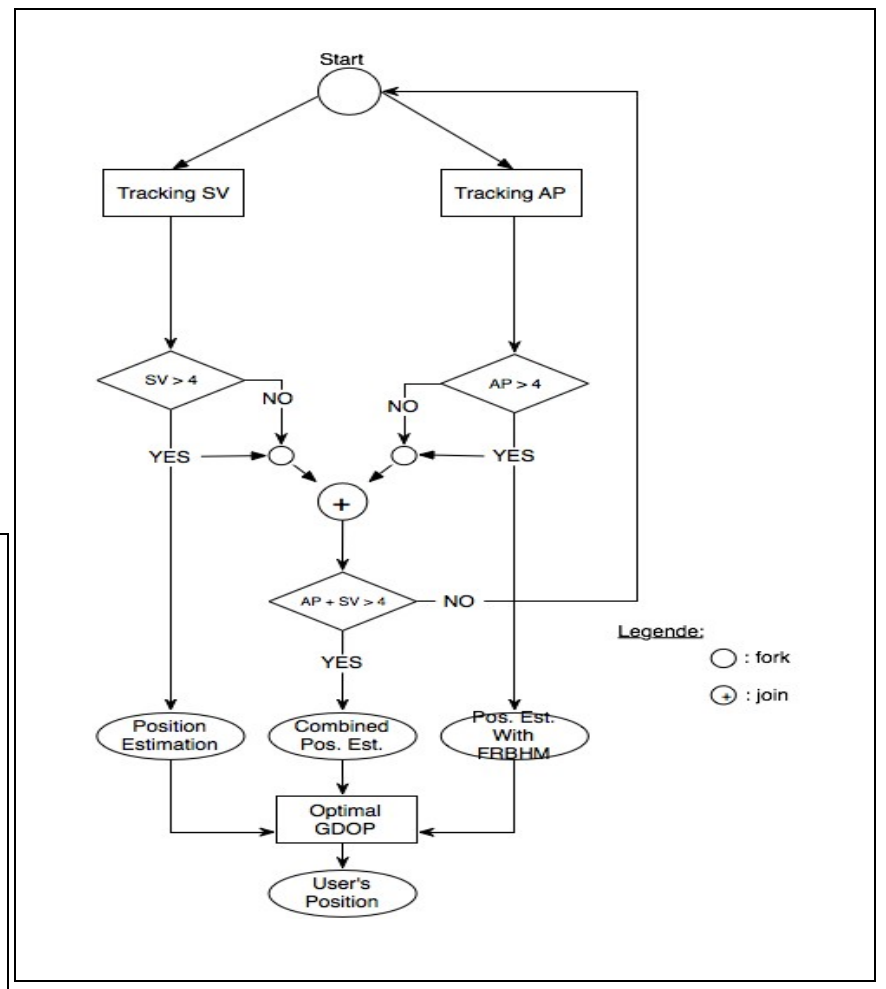

Figure 2. The algorithm of the combined position system based on GPS and Wi-Fi.

We initiate our algorithm with tracking signals from both AP and GPS satellites. If the number of visible satellites is less than four or the number of visible AP is less than four then we combine them so that the number of visible $\mathrm{AP}$ and satellites is more than four.

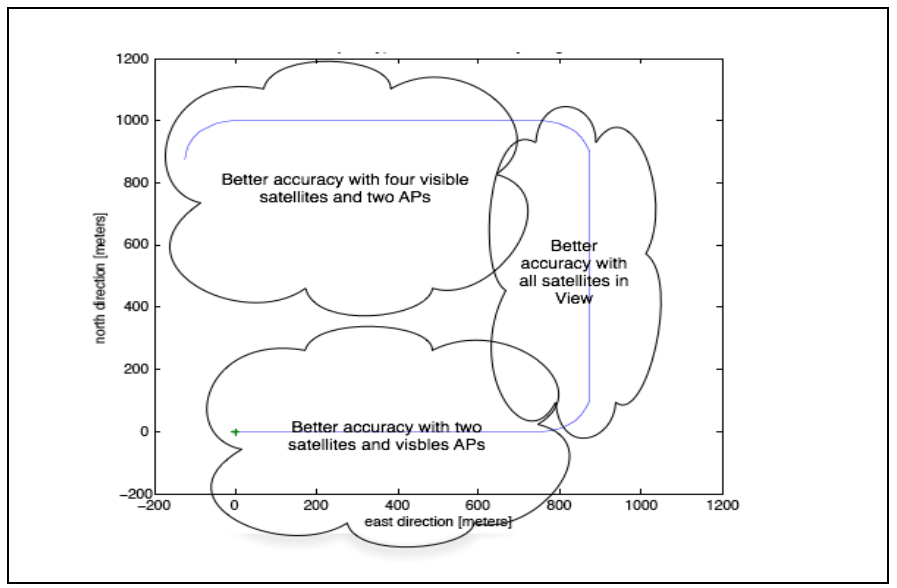

Figure 3. The accuracy depends on the configuration of the combined position system 
The accuracy depends on the combinations of measurements from satellites and APs (see Figure 3). This leads us to thinking: Why not switch configuration/combination to another while having a goal to achieve maximum accuracy? What is the indicator that allowed us to choose the configuration/combination ideal for a situation " $s$ " at time " $t$ "?

In our case, the more appropriate formulation of this GDOP that can help us to choose the best configuration of our combined position system at the time " $t$ " in the situation " $s$ " is the following:

$$
\begin{aligned}
& H=\left[\begin{array}{cccc}
a_{1, x} & a_{1, y} & a_{1, z} & 1 \\
\vdots & \vdots & \vdots & \vdots \\
a_{N_{S A T}, x} & a_{N_{S_{A T}, y}} & a_{N_{S A T}, z} & 1 \\
b_{1, x} & b_{1, y} & b_{1, z} & 0 \\
\vdots & \vdots & \vdots & \vdots \\
b_{N_{A P}, x} & b_{N_{A P}, y} & b_{N_{A P}, z} & 0
\end{array}\right] \\
& \text { where } a_{x i}=\frac{\left(X^{i}-\hat{X}_{u}\right)}{\hat{r}_{i}}, a_{y i}=\frac{\left(Y^{i}-\hat{Y}_{u}\right)}{\hat{r}_{i}} \text { and } a_{z i}= \\
& \frac{\left(Z^{i}-\hat{Z}_{u}\right)}{\hat{r}_{i}} \text {. }
\end{aligned}
$$

The GDOP is:

$$
G D O P=\sqrt{\operatorname{Tr}\left[\left(H^{T} H\right)^{-1}\right]}
$$

\section{Simulation}

This section presents some results from our proposal simulations. Performance of the position estimation technology was tested with different number of satellites in view and different number of Access Points in view.

The GPS measurements were combined with forward link measurements from at least one access point in situations where the number of satellites visible was not enough to compute a location.

The algorithm of our simulation follows the steps bellow:

1 we use the Kepler parameters for ideal circular orbits from the matrix in gpskep.mat and maintain as global variables with GpsSoft (1996)

2 We generate a path that consists of straight segments joined by constant-radius turns.

3 Generate matrix of positions of satellites which are

4 We generate the visible satellites to the user at location usrxyz.

5 We generate the visible Access Points to the user at location usrxyz.

6 We generate a vector of 'measured' pseudoranges and accumulated delta ranges (i.e., integrated Doppler) to all visible satellites.

7 We generate a vector of 'measured' distances and accumulated delta ranges (i.e., integrated Doppler) to all visible Access Points.
8 We compute position from satellite and Access Points positions, pseudoranges and distances via ordinary least squares.

The following curves present simulation results from each scenario.

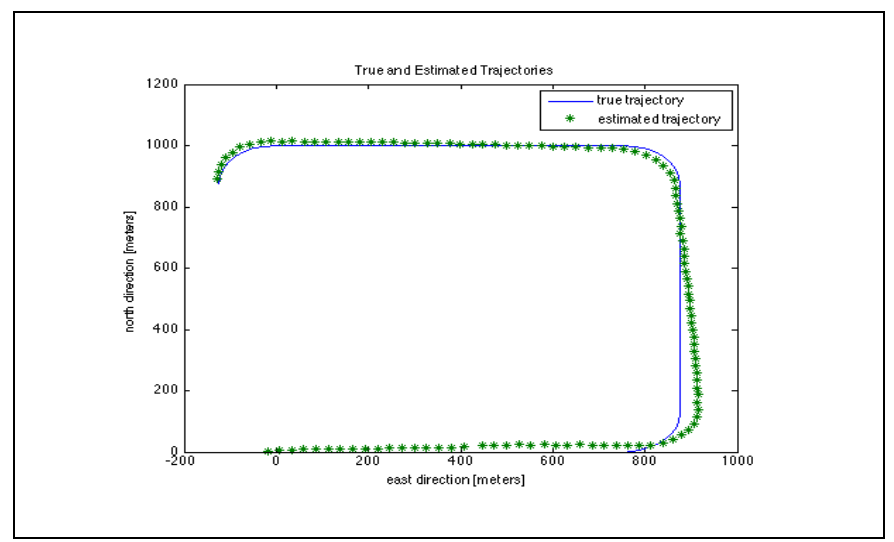

Figure 4. All satellites in view. The user is in an open environment. The selective availability is activated. Mean error $=36.4022 \mathrm{~m}$. The mean error is equal to $25 \mathrm{~m}$ when we use additional APs

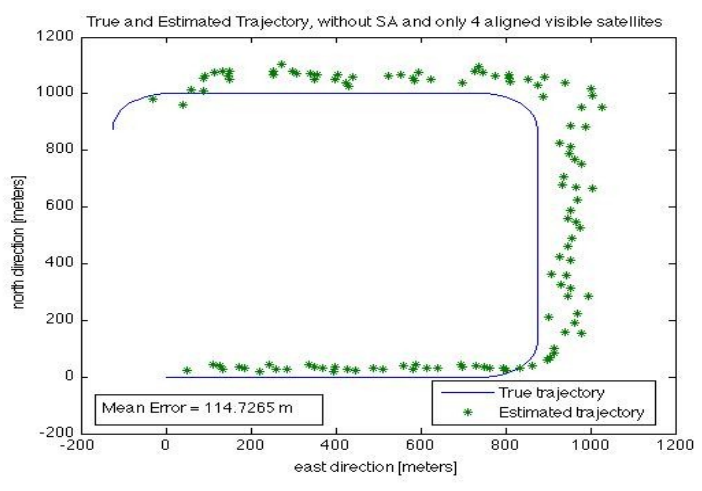

Figure 5. The true and the estimated trajectory with only four aligned satellites. The selective availability is disabled..

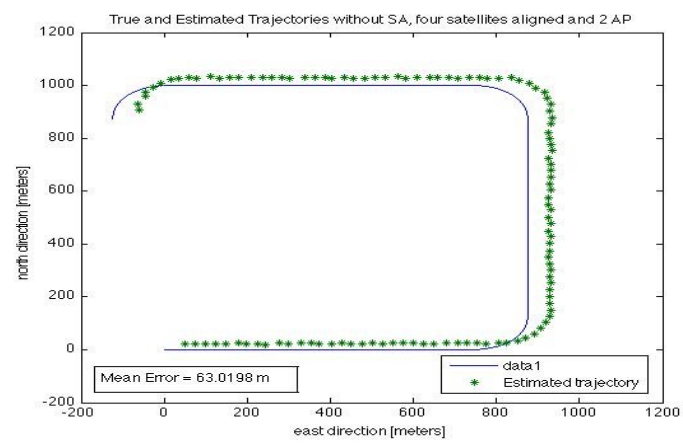


Figure 6. The true and the estimated trajectory with four aligned satellites and two APs. The selective availability is disabled..

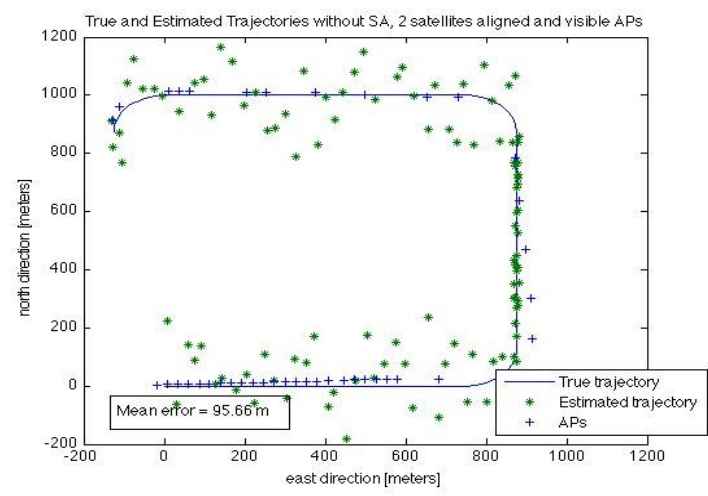

Figure 7. The true and the estimated trajectory with only one aligned satellites and visible APs. The selective availability is disabled.

\section{AnALYSIS}

After analyzing the various curves presented in the previous section, we conclude that the accuracy is best according to the various combinations of measurements from satellites and APs.

When we compare combined positionning system based on GPS and cellular networks with our proposition that combines GPS and Wi-Fi networks, we conclude that the accuracy of our proposition stay always under a threshold of 100 meter. This quality guaranty to the user the knowledge of an approximate location even if the condition deplorable. The Table 1. Shows that the estimated position with gpsOne can reach 345 meters in poor conditions such as only one satellite in view when, with the same conditions noted proposal remains below the 100 meter. This accuracy is sufficient in the case of application that seeks only indicate eg restaurants are near the user.

TABLE I. RESULTS COMPARISON

\begin{tabular}{|l|l|c|}
\hline & \multicolumn{1}{|c|}{ GpsOne } & Our proposal \\
\hline Four satellites & 18 meter & 19 meter \\
\hline Three satellites & 35 meter & 80 meter \\
\hline Two satellites & 253 méter & 78 meter \\
\hline One satellites & 345 meter & 95 meter \\
\hline
\end{tabular}

\section{Conclusion}

The simulation done, shows that the accuracy is less than 100 meter in the case of having two satellites and two Access Points in view instead of 253 meter [5] using gpsOne. The simulations shows also that when the number of satellites is equal to four or above, and when the angle of the satellites perception is unsatisfactory (satellites more or less aligned), a combined solution can make the positioning accuracy of about $5 \mathrm{~m}$, even environments poorly covered or not covered at all (indoor, urban canyons, etc.). Indeed, regardless of the environment, the user continues to have a position even if the accuracy exceeds one meter.

The main idea of our system is based on the principle of providing an idea about the location of the user at any time (even if the accuracy is not of the order of a meter) rather than the absence of any clue to the position.

\section{REFERENCES}

[1] S. Kyriazakos, D. Drakoulis, M. Theologou, J.-A. Sanchez-P., "Localization of Mobile Terminals, based on a Hybrid Satelliteassisted and Network-based Techniques", Proceedings WCNC, Chicago September 2000.

[2] Indoor Localisation - Technologies and Applications. Alexander Klapproth and Stefan Knauth. Lucerne University of Applied Sciences. CEESAR - Centre of Excellence for Embedded Systems Applied Research

[3] Navstar Global Positioning System Surveying.(1 july 2003). Michael j. WalshColonel Micheal J.Walsh

[4] GPS Theory and Practice (2001) B Hofmann-Wellenhof, H Lichtenegger, J Collins

[5] gpsOneTM: a hybrid position location system Soliman, S. Agashe, P. Fernandez, I. Vayanos, A. Gaal, P. Oljaca, M. QUALCOMM Inc., Spread Spectrum Techniques and Applications, 2000 IEEE Sixth International Symposium, Sep 2000. On page(s): 330-335 vol.1

[6] Hybrid Position Location Technology White Paper. gpsOne Overview . Qualcomm CDMA Technologies

[7] http://support.vzw.com/clc/features/data_services/vz_navigator.html

[8] Correcting GPS position in a hybrid navigation system, Wei-Wen Kao.

[9] Paramvir Bahl and Venkata N. Padmanabhan. RADAR : An inbuilding RF-based user location and tracking system. In INFOCOM (2), pages 775:784, 2000.

[10] R. Roos, P. Myllymaki, H. Tirri, P. Misikangas, and J. Sievänen. A Probabilistic Approach to WLAN User Location Estimation. International Journal of Wireless Information Networks, 9(3) :155164 , July 2002.

[11] F. Lassabe, O. Baala, Ph. Canalda, P. Chatonnay, and F. Spies. A friisbased calibrated model for WiFi terminals positioning. In Proceedings of IEEE Int. Symp. on a World Wireless, Mobile and Multimedia Networks (WoWMoM 2005), pages 382-387, Taormina, Italy, June 2005.

[12] http ://www.skyhookwireless.com.

[13] Soumaya Zirari, Philippe Canalda, and François Spies. Modelling and Emulation of an Extended GDOP For Hybrid And Combined Positioning System. In ENC-GNSS'09, European Navigation Conference - Global Navigation Satellite Systems, Naples, Italy, May 2009

[14] Soumaya Zirari, Philippe Canalda, and François Spies. A Very First Geometric Dilution Of Precision Proposal For Wireless Access Mobile Networks. In SPACOMM'09, 1st Int. Conf. on Advances in Satellite and Space Communications, Colmar, France, July 2009.

[15] http://www.astronautix.com/craft/timation.htm

[16] http://news.stanford.edu/pr/95/950613Arc5183.html 